\title{
Prevención de trastornos osteomioarticulares en el puesto de eviscerado en un Camal Municipal. Caso de la Ciudad de Ambato
}

Prevention of osteomyoarticular disorders in the evisceration station in a Municipal Slaughterhouse. Case of Ambato City

María Gabriela Romo Sunta. ${ }^{1}$, Manolo Alexander Córdova Suárez. ${ }^{2}$ \& Edison Patricio Villacres Cevallos. ${ }^{3}$

\begin{abstract}
.
Introduction. The slaughter of cattle is a semi-industrial work in sequence, where constant force is exerted and repetitive actions are carried out that can make this activity very dangerous to suffer from osteomyoarticular disorders, such as the job of an expert in the Municipal Chamber of the Decentralized Autonomous Government. (GAD) of the City of Ambato. Objective. This research designs an ergonomic position to mitigate distal affections in the upper extremities
\end{abstract}

\section{Resumen.}

Introducción. El faenamiento de ganado es un trabajo semi-industrial en secuencia, donde se ejerce fuerza constante y se ejecutan acciones repetitivas que puede hacer de esta actividad muy peligrosa para sufrir trastornos osteomioarticulares como es el puesto de trabajo de evicerado del Camal Municipal del Gobierno Autónomo Descentralizado (GAD) de la Ciudad de Ambato. Objetivo. Esta investigación diseña un puesto ergonómico para atenuar

\footnotetext{
1 Regional Autonomous University of Los Andes, Postgraduate, Ambato, Ecuador, pg.mariagrs60@uniandes.edu.ec, (D) https://orcid.org/0000-0002-2350-3191

2 Universidad Nacional de Chimborazo, Facultad de Ingeniería, Riobamba-Ecuador, manolo.cordova@unach.edu.ec (D) https://orcid.org/0000-0001-6786-7926

3 Universidad Nacional de Chimborazo, Facultad de Ingeniería, Riobamba-Ecuador, pvillacres@ unach.edu.ec (D) https://orcid.org/0000-0001-9518-1278
} 
due to the manipulation of viscera in a continuous process line and with efforts to adapt to fixed surfaces and humid and cold conditions that multiply the effort. Methodology. First, the Occupational Repetitive Action (OCRA) method was used to establish the important variables in the actions that are carried out while completing cycles and where perceptual or cognitive activities clearly prevail and movements of the upper limbs are residual. Then the possibility of implementing an adjustable platform was studied to improve posture and reduce the level of risk due to repetitive movement. To deepen the study, a controlled time plan was combined, movements until a significant decrease was achieved. Results. The results of the implementation of a new work platform show a $45 \%$ decrease in the OCRA index, considering: a) $23.3 \%$ decrease in time in shoulder position, $16.7 \%$ in elbow, use of heated gloves, elimination of clamping height, increased a break in the work day other than lunch, elimination of static clamping height, reduction of a technical action, a mirror post was added and lowered to a dynamic technical action with 20 actions in one minute. Conclusion. By placing the work plane anthropometrically with the use of an adjustable platform, the ergonomic risk due to repetitive movement was reduced to $45 \%$.

Keywords: Beef, OCRA, gutted, ergonomics. las afecciones distales en extremidades superiores por la manipulación de vísceras en una línea de proceso continuo y con esfuerzos de adaptación a superficies fijas y condiciones de humedad $\mathrm{y}$ frio que multiplican el esfuerzo. Metodología. Primero se utilizó el método Occupational Repetitive Action (OCRA) para establecer las variables importantes en las acciones que se realizan cumpliendo ciclos y donde las actividades perceptivas o cognitivas prevalecen claramente y los movimientos de los miembros superiores son residuales. Luego se estudió la posibilidad de implementar una plataforma ajustable para mejorar la postura y atenuar el nivel de riesgo por movimiento repetitivo. Para ahondar el estudio se combinó un plan controlado de tiempos, movimientos hasta alcanzar una disminución significativa. Resultados. Los resultados de la implementación de una nueva plataforma de trabajo muestran una disminución del $45 \%$ en el índice OCRA, considerando: a) disminución del tiempo en la posición del hombro del $23,3 \%, 16,7 \%$ en el codo, uso de guantes térmicos, eliminación de altura de sujeción, aumento de una pausa en la jornada de trabajo aparte de la del almuerzo, eliminación de altura de sujeción estática, diminución de una acción técnica, se añadió un puesto espejo y se bajó a una acción técnica dinámica con 20 acciones en un minuto. Conclusión. $\quad \mathrm{Al}$ ubicar antropométricamente el plano de trabajo con el uso de una plataforma ajustable se redujo al $45 \%$ el riesgo ergonómico por movimiento repetitivo.

Palabras claves: Camal, OCRA, eviscerado, ergonomía. 


\section{Introducción.}

El incremento de la población a nivel mundial y las mejoras constantes en los sistemas de producción de carne de ganado vacuno y porcino, obligan a los municipio locales que administran el faenamiento de ganado a mejorar sus sistemas de producción y estándares para ser más eficientes (Errecart, Lucero, \& Sosa, 2015). El Camal es un lugar donde se realizan las operaciones de sacrificio y faenado del ganado que se destina para el abasto público; dicho establecimiento recibe diferentes nombres de acuerdo con la región o país donde se ubica: Camal, Rastro, Matadero, Frigorífico, Matadero (Camacho Macedo, 2017).

Por otro lado los problemas relacionados al sacrificio y faenado de ganados en Ecuador se pueden resumir en los siguientes puntos: a) Instalaciones, b) Equipamiento, c) Aseguramiento de la calidad, d) almacenamiento, e) mal manejo de los residuos y f) pérdidas económicas por un alto ausentismo laboral (Lucio-Quintana et al., 2018). Las regulaciones relacionadas a las mejoras de las buenas prácticas de manufacturas en este campo son dos: el Decreto Ejecutivo N 1583 y el Decreto Ejecutivo N ${ }^{\circ} 3253$ (Loor Avendaño \& López Berni, 2015). Las implicaciones de su incumplimiento no solo generan una deficiente producción sino también se observa un ambiente laboral peligroso. La mayoría de las personas que faltan a sus labores en los camales sufren trastornos osteomioarticulares por el uso continuo de equipos y herramientas menores en las actividades del faenamiento post mortem en la zona de canales o denominada zona húmeda (Lascano Moreta, 2015).

Si bien es cierto los procesos antes y después del faenamiento del ganado siguen una línea de producción y normativa establecida por la Agencia Nacional de Regulación, Control y Vigilancia Sanitaria (ARCSA) del Ecuador no se considera la ergonomía como aspecto principal en la eliminación de las molestias osteomioarticulares que presentan los trabajadores. Los puestos de trabajo en un camal requieren personal entrenado y ejecución de actividades con levantamiento, traslado, manipulación, sujeción, hacimiento de cargas, instauración de fuerzas, uso de herramientas cortopunzantes, uso de pistolas neumáticas, sierras y demás, en una línea de producción por lotes diarios y con tiempos y movimientos restringidos que complican el panorama ergonómico (Suárez, Vasco, Ochoa, \& Puca, 2020) y aceleran la presencia de accidentes y enfermedades del trabajo, convirtiendo a esta actividad en una de las más peligrosas en su ejecución (Loor Avendaño \& López Berni, 2015).

La línea de producción en un camal municipal tiene puntos críticos por el control de tiempos y movimientos, y uno de los puestos que sufre las consecuencias del rigor de trabajo y las condiciones inadecuadas es el de eviscerado, ya que además de ocupar un puesto donde el personal se adapta a la antropometría de su entorno existe la exposición a movimientos repetitivos y el hacimiento temporal de carga estáticas(Casanova Párraga \& De la Cruz Moreta, 2017).

La postura que adopta el trabajador en el puesto de eviscerado depende de la altura de la plataforma y la inclinación del canal de circulación de agua y víscera que recibe el 
trabajador. Por lo que personas con medidas antropométricas diferentes de las del diseño original sufren de molestias tempranas por esta incomodidad y a la larga determinan enfermedades relacionadas con problemas de trastornos osteomioarticulares (Carrera Yaselga \& Valencia Pabón, 2011).

Para mitigar los efectos disergonómicos causados por estas posiciones inadecuadas y sobreesfuerzos determinados por el ritmo de trabajo en la zona de eviscerado que afectan al sistema osteomioarticular de los trabajadores se debe rediseñar el entorno de trabajo con fundamentos de ergonomía o simplemente adoptar ayudas antropométricas que ayuden a la adaptación del personal en el manejo y sujeción de las vísceras en el proceso de canales (Usiña Revelo, 2020).

Estas ayudas ergonómicas en ocasiones no se pueden efectuar ya que el rediseño involucra cambios en los tiempos de trabajo o modificaciones con una alta inversión que los administradores ven estas transformaciones como un gasto y complicaciones en su organización del trabajo por lo se sugiere cambios factibles y de fácil aplicación (Natarén \& Elío, 2004).

En la actualidad, el uso de métodos ergonómicos que consideran los factores que inciden el aparecimiento de trastornos osteomioarticulares garantizan los resultados antes de la aplicación real, evitando problemas posteriores con gastos innecesarios y sin resultados. Por lo que en este trabajo se utilizó el método OCRA para cuantificar la mejora del rediseño ergonómico. Este método considera la duración de la exposición y seis factores: a) tiempos, b) recuperación, c) frecuencia, d) postura, e) fuerza y f) adicionales (Dimate García, Rodríguez Romero, González Rincón, Pardo López, \& Garibello Cubillos, 2019).

Esta investigación utiliza la ergonomía de diseño de los puestos de trabajo con el uso de una plataforma adaptable para la mejorar el puesto de eviscerado tan importante en el faenamiento de ganado.

\section{Metodología}

\section{Diseño Ergonómico de puestos de trabajo.}

El diseño ergonómico se fundamenta en la aplicación de medidas correctivas a los factores desencadenantes de enfermedades ocupacionales considerando antropometría de diseño y las técnicas establecidas para cada factor de riesgo laboral estimado como crítico en estudios previos a la implantación (Flores, 2001).

La antropometría de diseño considera modificaciones factibles de los espacios de trabajo para mejorar alcances, posturas, ángulos biomecánicos, posiciones de extensión, flexión, abducción, que ayudarán a una mejor ubicación del ejecutante de la tarea peligrosa (Luengo, 2004). En esta investigación es recomendó la ubicación de una plataforma metálica ajustable mecánicamente a la posición ideal que requiere el trabajador en el puesto de eviscerado (Lite, García, \& del Campo, 2007). 


\section{Método Occupational Repetitive Action (OCRA).}

Este estudio empieza con el análisis de la condición de repetitividad que menciona la Norma UNE-N-1005-5 en el puesto de trabajo de eviscerado del Camal del Gad de Ambato, revisando que la actividad cumplen con: a) trabajo por ciclos, b) prevalencia de movimientos repetitivos en los miembros superiores, c) comprobación de presencia de: fuerzas, movimientos alta repetitividad, frecuencia de acciones técnicas y factores adicionales que complican la tarea (Latonda et al., 2010).

Luego este trabajo utilizó uno de los métodos de análisis ergonómico más importantes para el estudio de movimientos repetitivos que el método Occupational Repetitive Action (OCRA) o también llamado la lista de chequeo OCRA (Pina, 2016).

Este método toma en cuenta movimientos de tareas que se repiten en ciclos cortos y que involucran la realización de esfuerzos o movimientos rápidos de: a) grupos musculares, b) huesos, c) articulaciones, d) tendones, e) ligamentos y f) nervios de una parte del cuerpo, comúnmente de las extremidades superiores. Es decir, la repetitividad es una característica de la tarea que provoca que el trabajador que la desarrolla esté continuamente repitiendo el mismo ciclo de trabajo, acciones técnicas y movimientos (Ramos \& Martínez Zamudio, 2016).

Como resultado el OCRA arroja: los valores por factor y movimientos estereotipados además un resultado de porcentaje de la carga máxima soportable alcanzado en cada articulación (Codo, hombro, torso, cadera, rodilla, tobillo) con un resultado global del nivel de riesgo por movimiento repetitivo y el índice OCRA (Picazo \& de Miguel, 2003).

\section{Resultados y discusión}

\section{Resultados de Método Occupational Repetitive Action (OCRA).}

Para evaluar el puesto de eviscerado se realizó un análisis preliminar observacional de campo del puesto de trabajo para identificar los aspectos que solicita el método OCRA como entradas. Este análisis se realizó a todos los trabajadores que ocupan el puesto de eviscerado teniendo como resultados los valores de la tabla $\mathrm{N}^{\circ} 1$.

En al figura $\mathrm{N}^{\circ} 1$ se observa el puesto analizado.

Tabla 1. Evaluación con el Método OCRA

\begin{tabular}{lc}
\hline \multicolumn{1}{c}{ ITEM } & \multicolumn{1}{c}{ Valor } \\
\hline & Organización del tiempo de trabajo \\
\hline Tiempo de exposición & 480 minutos \\
Pausas oficiales & 10 minutos \\
Pausas no oficiales & 5 minutos \\
Duración almuerzo & 60 minutos \\
Duración de tareas no & 30 minutos \\
repetitivas & \\
\hline
\end{tabular}


Tabla 1. Evaluación con el Método OCRA (continuación)

\begin{tabular}{|c|c|}
\hline \multirow[t]{2}{*}{ ITEM } & \multirow[b]{2}{*}{ Periodos de recuperación } \\
\hline & \\
\hline Periodos de recuperación & $\begin{array}{l}1 \text { pausa de al menos } 10 \text { minutos, en turno de } 7 \text { horas (sin pausa } \\
\text { en el almuerzo), o sólo } 1 \text { pausa para el almuerzo en un turno } \\
\text { de } 8 \text { horas. }\end{array}$ \\
\hline \multicolumn{2}{|r|}{ Frecuencias } \\
\hline Tiempo del ciclo & 180 segundos \\
\hline Número de acciones Técnicas & 4 \\
\hline Tipo de Acciones & Dinámicas y estáticas \\
\hline Acciones Técnicas Estáticas & $\begin{array}{l}\text { Se sostiene un objeto durante al menos } 5 \text { segundos, } \\
\text { realizándose una o más acciones estáticas durante } 3 / 3 \text { del } \\
\text { tiempo de ciclo (o de observación). }\end{array}$ \\
\hline Acciones Técnicas & Los movimientos del brazo son bastante rápidos (más de 40 \\
\hline Dinámicas & $\begin{array}{l}\text { acciones/minuto). Solo se permiten pequeñas pausas } \\
\text { ocasionales e irregulares }\end{array}$ \\
\hline \multicolumn{2}{|r|}{ Posturas } \\
\hline Posición del hombro & $\begin{array}{l}\text { Se mantienen a la altura de los hombros y sin soporte más o } \\
\text { menos el } 1 / 3 \text { del tiempo. }\end{array}$ \\
\hline Posición del codo & Movimientos repentinos más de la mitad del tiempo \\
\hline Posición de la Muñeca & $\begin{array}{l}\text { Doblada en una posición extrema o adoptada postura } \\
\text { forzadas al menos } 1 / 3 \text { del tiempo. }\end{array}$ \\
\hline Posición de la mano & Los dedos están en forma de gancho. 1/3 del tiempo. \\
\hline Movimientos estereotipados & $\begin{array}{l}\text { Repetición de movimientos idénticos del hombro, codo, } \\
\text { muñeca, o dedos casi todo el tiempo (o el tiempo de ciclo es } \\
\text { inferior a } 8 \text { segundos) }\end{array}$ \\
\hline \multicolumn{2}{|r|}{ Fuerza } \\
\hline \multirow[t]{2}{*}{ Actividades esfuerzo } & Cerrar o abrir, con fuerza moderada $1 / 3$ del tiempo \\
\hline & Utilizar herramientas, con fuerza moderada $1 / 3$ del tiempo \\
\hline \multicolumn{2}{|l|}{ Factores de riesgo adicionales } \\
\hline Frío & Exposición al frio más de la mitad del tiempo. \\
\hline Ritmo de trabajo & $\begin{array}{l}\text { Está parcialmente determinado por la máquina, con ritmo de } \\
\text { trabajo donde puede disminuirse o acelerarse }\end{array}$ \\
\hline \multicolumn{2}{|r|}{ Resultado final OCRA } \\
\hline & 21,3 \\
\hline
\end{tabular}

Nota: Los valores de cada factor son resultado de la observación de campo. 


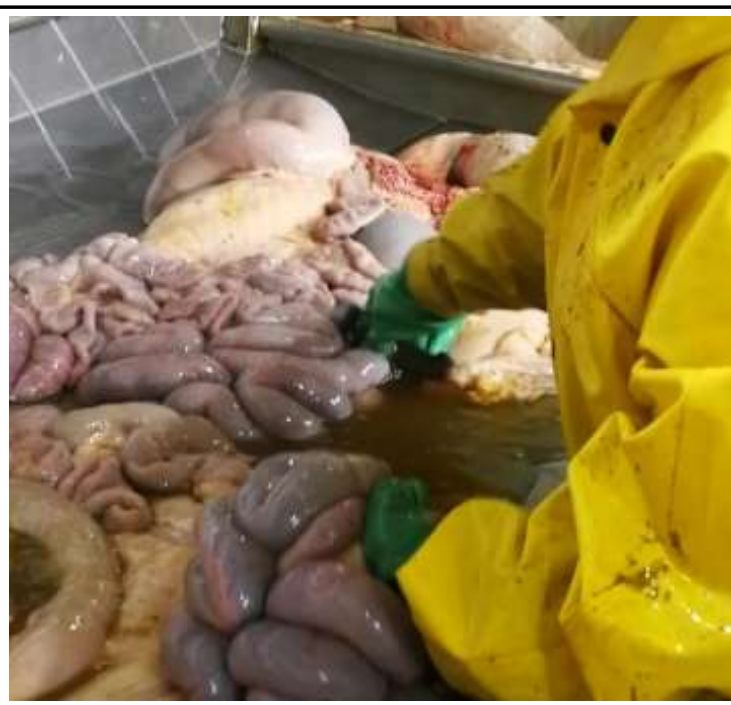

Figura 1. Puesto de trabajo de eviscerado. Nota: Adaptado UGR-GADMA.

Luego de implementar las medidas de prevención ergonómicas se obtiene los resultados de la tabla 2.

En la figura 2. Se observa los bípedos de las posturas del cuerpo antes y después de implementar las medidas de control y la adaptación de la plataforma móvil.
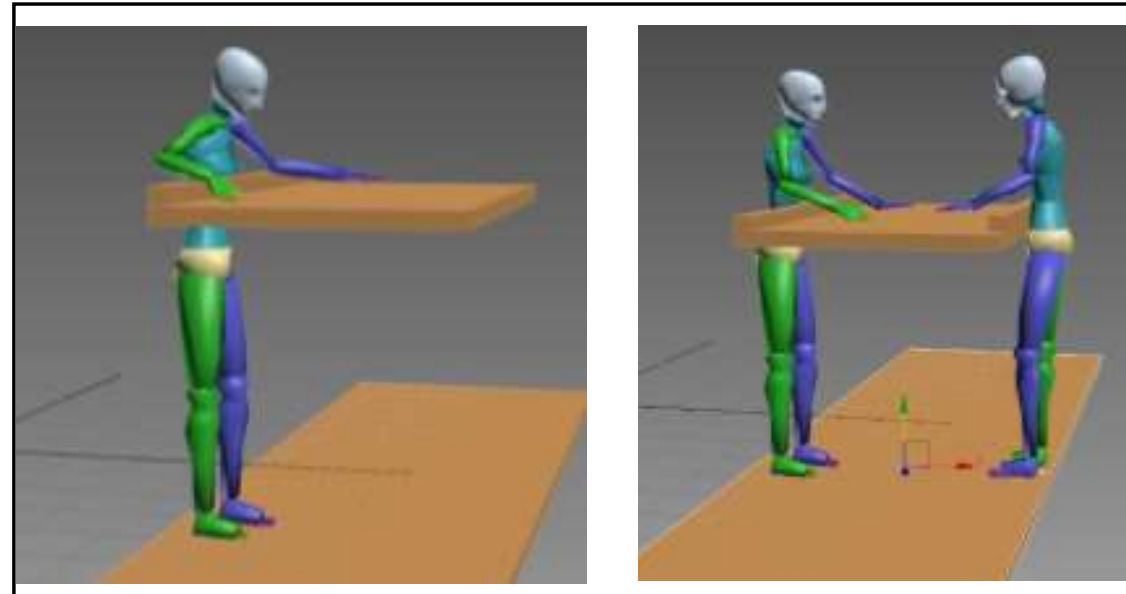

Figura 2. Adaptación de la plataforma de trabajo

Nota: Para el diagnóstico se bajó la plataforma de trabajo $20 \mathrm{~cm}$.

Tabla 2. Evaluación con el Método OCRA con modificaciones

\begin{tabular}{cc}
\hline ITEM & Valor \\
\hline \multicolumn{1}{c}{ Organización del tiempo de trabajo } \\
\hline Tiempo que el trabajador ocupa el puesto & 480 minutos \\
en la jornada & \\
Pausas oficiales & 20 minutos \\
Pausas no oficiales & 5 minutos \\
Duración del descanso para el almuerzo & 60 minutos \\
Duración de tareas no repetitivas & 30 minutos \\
\hline
\end{tabular}


Tabla 2. Evaluación con el Método OCRA con modificaciones (continuación)

\begin{tabular}{|c|c|}
\hline ITEM & Valor \\
\hline \multicolumn{2}{|c|}{ Periodos de recuperación } \\
\hline Periodos de recuperación & $\begin{array}{l}\text { Hay } 2 \text { pausas en un turno de seis horas o } 3 \text { pausas } \\
\text { en un turno de } 7 \text { a } 8 \text { horas }\end{array}$ \\
\hline \multicolumn{2}{|r|}{ Frecuencias } \\
\hline Tiempo del ciclo & 180 segundos \\
\hline Número de acciones Técnicas & 1 \\
\hline $\begin{array}{l}\text { Tipo de Acciones Técnicas más } \\
\text { representativas }\end{array}$ & Dinámicas y estáticas \\
\hline Acciones Técnicas Estáticas & $\begin{array}{c}\text { Se sostiene un objeto durante al menos } 5 \\
\text { segundos consecutivos, realizándose una o más } \\
\text { acciones estáticas durante } 2 / 3 \text { del tiempo de ciclo } \\
\text { (o de observación) }\end{array}$ \\
\hline Acciones Técnicas Dinámicas & $\begin{array}{c}\text { Los movimientos del brazo son bastante rápidos } \\
\text { (más de } 20 \text { acciones/minuto). Solo se permiten } \\
\text { pequeñas pausas ocasionales e irregulares }\end{array}$ \\
\hline \multicolumn{2}{|r|}{ Posturas } \\
\hline Posición del hombro & $\begin{array}{c}\text { Los brazos no poseen apoyo y permanece } \\
\text { ligeramente elevado algo más de la mitad del } \\
\text { tiempo }\end{array}$ \\
\hline Posición del codo & $\begin{array}{l}\text { El codo realiza movimientos repentinos (flexión - } \\
\text { extensión, o pronosupinación extrema, tirones, } \\
\text { golpes) al menos un tercio del tiempo }\end{array}$ \\
\hline Posición de la Muñeca & $\begin{array}{c}\text { La muñeca permanece doblada en una posición } \\
\text { extrema o adoptada postura forzadas (alto grado } \\
\text { de flexión-extensión o desviación lateral) al } \\
\text { menos } 1 / 3 \text { del tiempo. }\end{array}$ \\
\hline Posición de la mano & $\begin{array}{l}\text { Los dedos están en forma de gancho (agarre en } \\
\text { gancho). Alrededor de } 1 / 3 \text { del tiempo. }\end{array}$ \\
\hline Movimientos estereotipados & No se realizan movimientos estereotipados. \\
\hline \multicolumn{2}{|r|}{ Fuerza } \\
\hline Actividades que implican esfuerzo & $\begin{array}{c}\text { Cerrar o abrir, con fuerza moderada } 1 / 3 \mathrm{del} \\
\text { tiempo } \\
\text { Utilizar herramientas, con fuerza moderada } 1 / 3 \\
\text { del tiempo }\end{array}$ \\
\hline \multicolumn{2}{|c|}{ Factores de riesgo adicionales } \\
\hline $\begin{array}{c}\text { Frío } \\
\text { Ritmo de trabajo }\end{array}$ & $\begin{array}{l}\text { Se proporcionó guantes térmicos } \\
\text { Se puso ayuda de otra persona }\end{array}$ \\
\hline \multicolumn{2}{|r|}{ l OCRA } \\
\hline
\end{tabular}

Nota: Los valores de cada factor son resultado de la observación de campo.

\section{Conclusiones}

- Los resultados de la implementación de una plataforma de trabajo móvil (plano de trabajo) muestran una disminución del $45 \%$ en el índice OCRA $(21,3$ 9,8). Como resultado de la aplicación de las medidas de control ergonómicas se logró: la disminución del tiempo en la posición del hombro del 23,3\%, la disminución del tiempo en la posición del codo del $16,7 \%$, la eliminación de la altura de sujeción, el aumento de una pausa en la jornada de trabajo aparte de la del 
almuerzo, la eliminación de la altura de sujeción estática, la disminución de una acción técnica, La ejecución de una acción técnica dinámica de 20 acciones en un minuto. Además, se aumentó una persona en el puesto de trabajo para compartir la ejecución de las actividades.

\section{Referencias bibliográficas.}

Camacho Macedo, M. S. (2017). Compostaje de subproductos derivados del sacrificio y faenado de ganado del camal municipal de Huaraz, utilizando diferentes sustratos.

Carrera Yaselga, M. D. R., \& Valencia Pabón, D. F. (2011). Factores relacionados con las enfermedades ocasionadas por el contacto con los desechos sólidos y líquidos que tienen los trabajadores de las empresas de faenamiento de la Provincia de Imbabura en el periodode enero a octubre 2010.

Casanova Párraga, R. F., \& De la Cruz Moreta, E. F. (2017). Propuesta de mecanismos de prevención de riesgos laborales para la empresa pública municipal del Camal de Quevedo. Quevedo: UTEQ,

Dimate García, A. E., Rodríguez Romero, D. C., González Rincón, E. Y., Pardo López, D. M., \& Garibello Cubillos, Y. J. N. P. C. e. C. B. (2019). Método OCRA en diferentes sectores productivos. Una revisión de la literatura, 2007-2018. 17(31).

Errecart, V., Lucero, M., \& Sosa, M. J. R. d. h. w. u. e. a. e. e. e. r. C. (2015). Análisis del mercado mundial de carnes. 20.

Flores, C. (2001). Ergonomía para el diseño: Designio Teorpia y práctica.

Lascano Moreta, A. M. (2015). El sobreesfuerzo y su incidencia en los trastornos músculo esqueléticos de extremidades superiores de los trabajadores del Camal Frigorífico Municipal de Ambato. Universidad Técnica de Ambato. Dirección de Posgrado. Facultad de Ingeniería ...,

Latonda, L. T., Moreno, C. C., Jaén, J. D. G., González, F. M., Pastor, A. O., \& Folgado, R. R. J. R. d. b. (2010). Nueva versión 8.0 de la aplicación Ergo/IBV para la evaluación de riesgos ergonómicos y psicosociales. (53), 55-58.

Lite, A. S., García, M. G., \& del Campo, M. Á. M. (2007). Métodos de evaluación y herramientas aplicadas al diseño y optimización ergonómica de puestos de trabajo. Paper presented at the XI Congreso de Ingeniería de Organización.

Loor Avendaño, K. E., \& López Berni, M. B. (2015). Elaboración de una Guía Metodológica para Implementación del Reglamento de Buenas Prácticas de Manufactura según Decreto Ejecutivo 3253 en una Empresa de servicio de Catering Industrial en la Ciudad de Guayaquil. Universidad de Guayaquil, Facultad de Ingeniería Química., 
Lucio-Quintana, A. B., Yánez-Silva, D., Cortés, C., Erazo, T., Rodríguez, J., Rodríguez, J., \& Donato-Lucio, J. J. R. d. I. T. (2018). SANIDAD ANIMAL Y BIOSEGURIDAD EN EL FAENAMIENTO DE BOVINOS DEL CAMAL MUNICIPAL DE GUARANDA, PERÍODO 2016-2017. 2018, 277-282.

Luengo, M. H. (2004). Antropometría y diseño. In: Universidad de los Andes.

Natarén, J. J., \& Elío, M. N. J. S. d. 1. T. (2004). Los trastornos musculoesqueléticos y la fatiga como indicadores de deficiencias ergonómicas y en la organización del trabajo. 12(2), 27-41.

Picazo, A. R., \& de Miguel, J. L. (2003). NTP 629: Movimientos repetitivos: métodos de evaluación Método OCRA: actualización.

Pina, M. J. B. (2016). Método de evaluación ergonómica de tareas repetitivas, basado en simulación dinámica de esfuerzos con modelos humanos. Universidad de Zaragoza,

Ramos, J. E., \& Martínez Zamudio, G. (2016). Evaluación de movimientos repetitivos en el proceso de fabricación de shampoo mediante la metodología Check List OCRA.

Suárez, M. A. C., Vasco, L. J. T., Ochoa, Z. E. Z., \& Puca, J. P. M. J. C. (2020). Diseño biomecánico del puesto de trabajo de noqueo en el faenamiento de ganado. 3(3.2), 6-17.

Usiña Revelo, A. P. (2020). Evaluación de riesgos ergonómicos asociados a lesiones músculo-esqueléticas en el área de faenado de ganado mayor del Camal Frigorífico Municipal de Ambato (CFMA). Universidad Técnica de Ambato. Facultad de Ingeniería en Sistemas ...,

\section{LCiencia}




\section{PARA CITAR EL ARTÍCULO INDEXADO.}

Romo Sunta, M. G., Córdova Suárez, M. A., \& Villacres Cevallos, E. P. (2021). Prevención de trastornos osteomioarticulares en el puesto de eviscerado en un Camal Municipal. Caso de la Ciudad de Ambato . Anatomía Digital, 4(3.1), 87-97. https://doi.org/10.33262/anatomiadigital.v4i3.1.1863

\section{Ciencia \\ Ligital}

El artículo que se publica es de exclusiva responsabilidad de los autores y no necesariamente reflejan el pensamiento de la Revista Anatomía Digital.

El artículo queda en propiedad de la revista y, por tanto, su publicación parcial y/o total en otro medio tiene que ser autorizado por el director de la Revista Anatomía Digital.
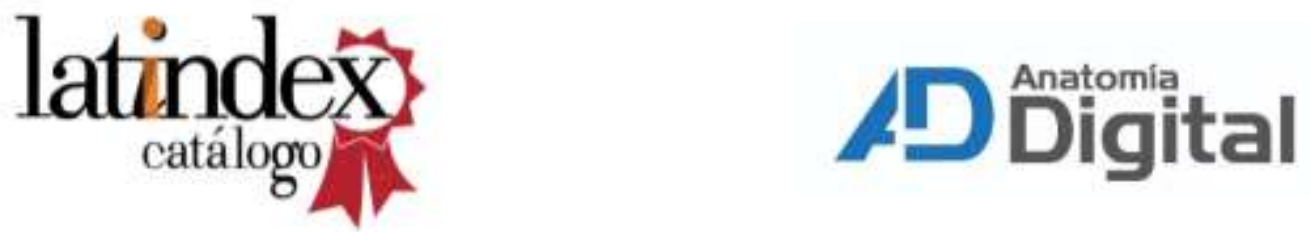